\title{
Pleuritic Pain
}

National Cancer Institute

\section{Source}

National Cancer Institute. Pleuritic Pain. NCI Thesaurus. Code C78564.

Painful sensation in the pleura. 